\title{
Properties and Processing of Cork Powder Filled Cellulose Derivatives Composites
}

Maria Helena Godinho ${ }^{1}$, Assis Farinha Martins ${ }^{1}$, Mohamed Naceur Belgacem ${ }^{2 *}$, Luís Gil ${ }^{3}$, Nereida Cordeiro ${ }^{4}$

${ }^{1}$ Dept. Ciência dos Materiais and CENIMAT, FCTIUNL, P-2825 Monte da Caparica, Portugal

${ }^{2}$ Dept Ciência e Tecnologia do Papel, Universidade da Beira Interior, P-6200

Covilhã, Portugal

${ }^{3}$ UTMC, INETI, Estrada das Palmeiras, Queluz de Baixo, P-2745 Barcarena, Portugal

${ }^{4}$ Dept Química, Universidade da Madeira, P-9000 Funchal, Portugal

SUMMARY: This paper deals with the use of cork powder, a by-product from cork industry, as a filler to reinforce hydroxypropyl cellulose (HPC) matrix. Several films were prepared using HPC, as a matrix, filled with different amounts of cork powder (average diameter $<50 \mu \mathrm{m})(0.0 ; 0.5 ; 1.0$ and $10.0 \% \mathrm{w} / \mathrm{w})$ and in the presence or not of 1,4-diisocyanatobutane (BDI) $(7.0 \% \mathrm{w} / \mathrm{w})$. Before the elaboration of these films, the surface properties of cork powder as well as that of suberin (main component of cork) were determined by Inverse Gas Chromatography (IGC). The tensile properties of the solid films obtained were studied and, as expected, for the films with BDI but without cork powder, the Young's modulus and the tensile strength increased, while the elongation decreased. However with the filled films it seemed that the Young's modulus decreased and the elongation increased. The Scanning Electron Microscopy showed that the fractured plane of samples with cross-linking agent and cork powder displayed some nucleation points $(0.3 \mu \mathrm{m})$ which indicates a strongly bonded interface and which could be considered as a responsible for the high mechanical properties observed.

\section{Introduction}

Portugal produces about $55 \%$ of the world-wide quantity of cork (Quercus suber L.). The transformation of this renewable material with unique properties into different commodities yields a large amount (up to $25 \%$ by weight) of solid waste having small particle size without 
industrial interest. Only a part of this by-product is valorised in the area of agglomerated panels, the rest is usually burned for energy recovering. Recently, original papers dealing with a comprehensive research program involving a preliminary study of cork and its components in order to propose more rational ways of using this wastes were reported in the literature ${ }^{1-4)}$. These studies dealt with cork itself but also with its major component: suberin which was studied extensively ${ }^{1-4)}$. This piece of work is an extension of the previous studies and it deals with the use of cork powder as a filler for thermoplastic matrices.

In order to obtain filled thermoplastic films with optimal properties, the surface properties of the filler and that of the matrix should be ascertained. The surface properties of cork were determined $^{5)}$ by contact angle measurements and found to be about $32 \mathrm{~mJ} / \mathrm{m}^{2}$. The major component of this energy had a dispersive character, i.e. $24 \mathrm{~mJ} / \mathrm{m}^{2}$. It is well known that the contact angle measurements showed serious limitations when applied to rough and porous material which is the case of cork. Recently, Inverse Gas Chromatography ${ }^{6}$ was found to be suitable technique in characterising the surfaces of fibers, such as, aramid, glass, carbon and cellulose or powders like silicas or activated carbons.

The main objective of this work is to report results concerning the elaboration of filled elastomers, based on hydroxypropyl cellulose as a matrix, cork as a reactive filler and 1,4diisocyanatobutane (BDI), as a coupling agent. Before reporting these data, the results of characterisation of cork and suberin by IGC will be discussed.

\section{Experimental Part}

\section{Material}

The HPC was of commercial reagent grade-Aldrich, $\mathrm{Mw}=100000 \mathrm{~g} \cdot \mathrm{mol}^{-1}$. The cross-linking agent (BDI) and the solvent (acetone) were purchased from Aldrich and used as received. Commercial granulated cork $(2-3 \mathrm{~mm})$ was ground on a Retsch mill with a sieve of $0.25 \mathrm{~mm}$. The fraction with particle sizes smaller than $50 \mu \mathrm{m}$ was selected for the experiments.

\section{Film Preparation}

A homogeneous solution (10-20\% w/w) was prepared by mixing HPC polymer with acetone. The mixture was then stored in the absence of day light with a continuous stirring for about 
one week. This solution was divided into four aliquots. One of them was spread on a flat Teflon film, and the solvent was allowed to evaporate for about 4 days in a laboratory atmosphere $\left(\mathrm{T}=20^{\circ} \mathrm{C}\right)$, a cross-linked film was also cast from this solution by mixing, under nitrogen atmosphere, with BDI $(7 \% \mathrm{w} / \mathrm{w})$. The rest of the solution was divided into different parts and an increasing amount of cork powder was added, namely: 0.5, 1.0 and 10.0\% w/w. The solutions were kept under stirring and in the absence of day light for about 2 weeks. Then, solid films were cast from these solutions. Cross-linked films were also prepared from the solutions with cork powder and BDI. The films were stored in a dessicator at room temperature.

\section{Characterisation}

The IGC equipment and conditions, the molecule probes as well as the column preparation and conditioning were described in previous studies ${ }^{2,3)}$. Some of these films were characterised by Scanning Electron Microscopy (SEM) using a SEM DSM962 model from Zeiss Company. The films were fractured under cryogenic conditions using liquid nitrogen. The tensile measurements were carried out on a Rheometric Scientific (Minimat - Firmware Vsn 3.1) Model, at room temperature $\left(23^{\circ} \mathrm{C}\right)$. Samples were cut into pieces with $5 \times 2 \mathrm{~cm}$. Five to ten successful determinations were made and the average value was calculated. The stretching rate was $5 \mathrm{~mm} / \mathrm{min}$.

\section{Results and Discussion}

Before starting the elaboration of the filled elastomers, the surface properties of cork and its main component, i.e. suberin were studied ${ }^{2,3)}$. As shown from Table 1, the London component of the surface energy was found 41 and $48 \mathrm{~mJ} / \mathrm{m}^{2}$, for cork and suberin, respectively.

Table 1. The dispersive and acid-base properties of cork and its main component: suberin.

\begin{tabular}{cccc}
\hline Material & Equation, $\gamma_{\mathbf{S}}^{\mathbf{D}}-\left(\mathbf{d} \gamma_{\mathbf{S}}^{\mathbf{D}} / \mathbf{d T}\right)^{*} \mathbf{T}$ & $\gamma_{\mathbf{S}} \mathbf{D}$, at $25^{\circ} \mathrm{C}, \mathrm{mJ} / \mathrm{m}^{2}$ & $\mathbf{K}_{\mathbf{A}} / \mathbf{K}_{\mathbf{B}}$ \\
Cork & $46.6-0.22 \mathrm{~T}$ & 41.1 & 1.1 \\
Suberin & $52.0-0.16 \mathrm{~T}$ & 48.0 & 2.3 \\
\hline
\end{tabular}

The dispersive component of the surface energy of cork was higher than that reported in the literature $^{5)}$, as determined using contact angle measurements. The dispersive contribution to 
the surface energy of suberin was also slightly higher than the value found using Wilhelmy plate and maximum bubble pressure measurements ${ }^{3)}$. This phenomenon was already observed for other materials, such as cellulose ${ }^{7)}$ and was attributed to the fact that IGC at infinite dilution is very sensitive to high energy sites of the material under study, which gives systematically higher values of dispersive energy. This is due to the heterogeneity of the solid surfaces. The acid-base properties of cork surface showed that it has an amphoteric character which should make it compatible with both acidic and basic matrices. Suberin displayed slightly acidic surface probably because it contains higher content of hydroxylic, carboxylic and ester groups.

The mechanical properties of the films obtained were determined and Figure 1 shows typical stress-strain curves for some samples. The data of the mechanical properties of these filled elastomers were deduced from these curves and are resumed in Table 2, from which some concluding remarks could be drawn, namely: (i) as expected, the use of cross-linking agents increased the Young's modulus and the tensile strength of the HPC matrix, while the elongation decreased. This can be easily seen if one compares films \#1 and \#5. (ii) the use of cork as a filler, i.e. in the absence of cross-linking agent (samples \#1 to 4), induced an increase of the Young's modulus and tensile strength and decreased the elongation of these films, (iii) the addition of $0.5 \% \mathrm{w} / \mathrm{w}$ of cork into films without cross-linking agent seemed to be the optimal amount introduced, since it gave the maximum increase of the Young's modulus and the tensile strength, as the case of film \#2. This could be attributed to the better dispersion of $0.5 \%$ of cork powder in comparison to that observed when 1 or $10 \%$ of the filler was added, and (iv) finally, the use of cork as a reactive filler, i.e., in the presence of crosslinking agent, gave a slight decrease of the Young's modulus and an increase of the elongation. These results are very promising since they provide a simple way to improve the stiffness of HPC and the elongation of HPC cross-linked films.

The films were also characterised by Scanning Electron Microscopy in order to evaluate the quality of the interface between cork particles and HPC matrix. In fact, two films containing the same amount of cork were elaborated. The first one was prepared without cross-linked agent whereas the second contained $7 \%$ of BDI. As shown from Photo 1, the film containing BDI showed nucleation points sizing about $0.3 \mu \mathrm{m}$ (see Photo $1 \mathrm{~b}$ ). This phenomenon was not observed for the film prepared without BDI (see Photo 1a). This observation is of very great importance, since it permitted to deduce that the interface cork-BDI-HPC seemed to be well 
bonded, which allowed to conclude that HPC/cork powder are chemically compatible.

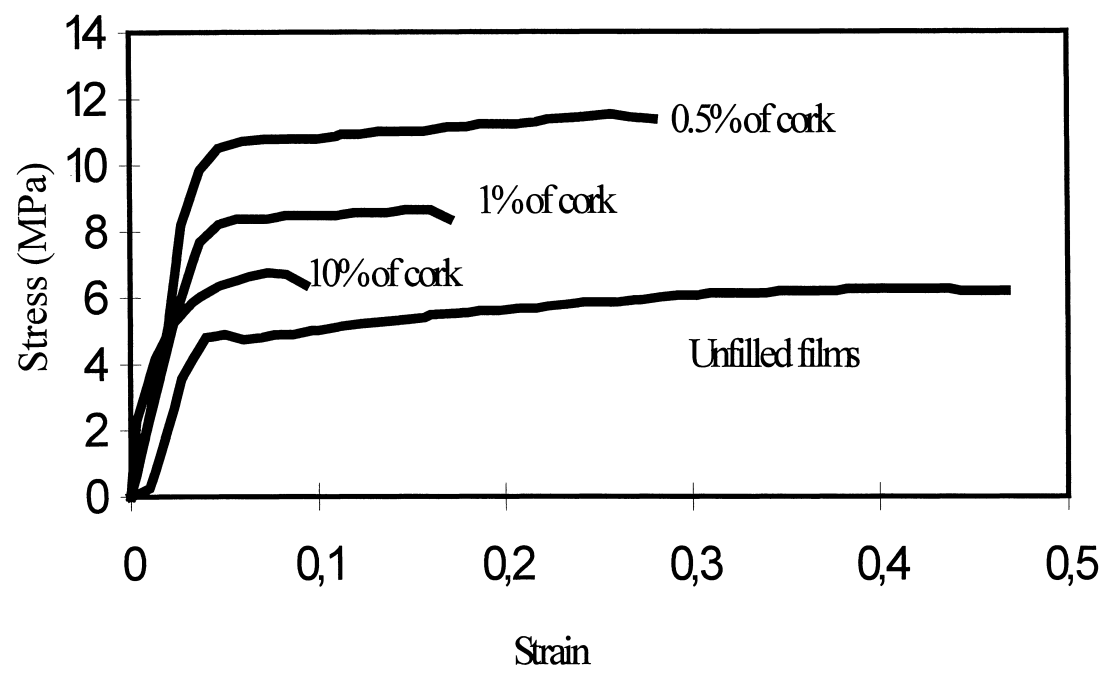

Fig. 1: Stress-strain curves cork powder filled HPC without BDI (see table 2).

Table 2. Tensile properties of cork filled HPC films.

\begin{tabular}{|c|c|c|c|c|c|c|}
\hline \multicolumn{3}{|c|}{ Sample } & \multirow{2}{*}{$\begin{array}{c}\text { Thickness } \\
(\mu \mathrm{m})\end{array}$} & \multirow{2}{*}{$\begin{array}{l}\text { Young's } \\
\text { Modulus } \\
\left(10^{8} \mathrm{~Pa}\right)\end{array}$} & \multirow{2}{*}{$\begin{array}{l}\text { Tensile } \\
\text { Strength } \\
\left(10^{6} \mathrm{~Pa}\right)\end{array}$} & \multirow{2}{*}{$\begin{array}{c}\text { Elongation } \\
\qquad \begin{array}{c}(\varepsilon) \\
(\%)\end{array}\end{array}$} \\
\hline $\begin{array}{l}\text { Cork } \\
(\% \mathrm{w} / \mathrm{w})\end{array}$ & $\begin{array}{c}\text { Cross-linking. } \\
\text { Agent } \\
(\mathrm{w} / \mathrm{w})\end{array}$ & Number & & & & \\
\hline 0.0 & 0.0 & $\# 1$ & 76 & 1.57 & 6.18 & 147 \\
\hline 0.5 & 0.0 & $\# 2$ & 69 & 2.77 & 11.38 & 128 \\
\hline 1.0 & 0.0 & $\# 3$ & 56 & 2.09 & 8.39 & 117 \\
\hline 10.0 & 0.0 & $\# 4$ & 83 & 2.06 & 6.38 & 109 \\
\hline 0.0 & 7.0 & $\# 5$ & 49 & 2.34 & 11.53 & 111 \\
\hline 0.5 & 7.0 & $\# 6$ & 71 & 2.00 & 12.32 & 133 \\
\hline 1.0 & 7.0 & $\# 7$ & 61 & 1.57 & 11.23 & 130 \\
\hline
\end{tabular}

This finding corroborates very well with the results obtained by IGC showing that HPC and cork are energetically compatible and the fact that it is suitable for both acidic and basic matrices. Moreover, these performances are not surprising if one considers that the components of cork are $\mathrm{OH}$-rich structures. 


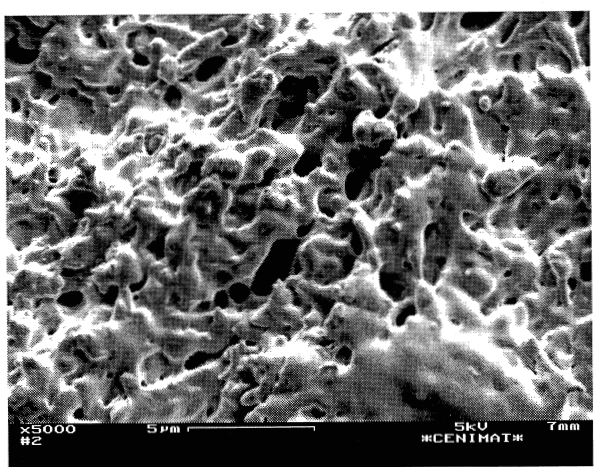

a)

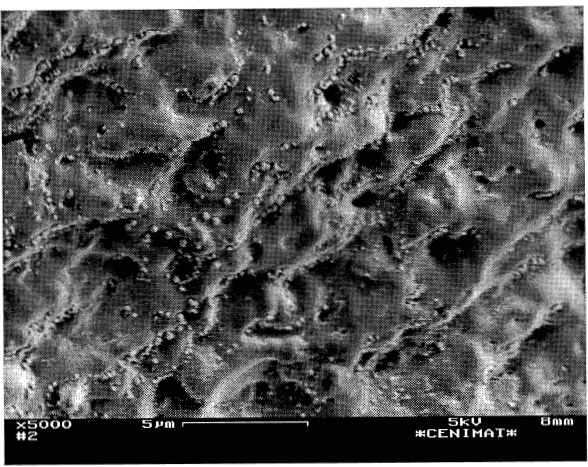

b)

Photo 1. Scanning electron photomicrograph of the fractured plane for ; a) sample with $0.5 \%$ w/w of cork without BDI; b) same sample with BDI.

\section{Conclusions}

This work is a very good introduction for cork filled films since it shows very promising results of surface compatibility between this by-product and HPC. It gives one more potential use of this waste. It is worth noting that both filler and matrix come from vegetal biomass, which is biodegradable and renewable.

\section{References}

1. Pascoal Neto, C., Cordeiro, N., Seca, A., Domingues, F., Gandini, A., Robert, D., Holzforschung 50, 563 (1996)

2. Cordeiro, N., Pascoal Neto, C., Gandini, A., Belgacem, M. N., J. Colloid Interface Sci. 174, 246 (1995)

3. Cordeiro, N., Aurenty, P., Belgacem, M. N., Gandini, A., Pascoal Neto, C., J. Colloid Interface Sci. 187, 498 (1997)

4. Cordeiro, N., Belgacem, M. N., Gandini, A., Pascoal Neto, C., Int. J. Biol. Macromol. 22, 71 (1998)

5. Gomes, C. S., Fernandes, A. C. and Almeida B. J. S., J. Colloid Interface Sci., 156, 196 (1993)

6. Belgacem, M. N. and Gandini, A., in Interfacial Phenomena in Chromaography, (E. Pefferkorn, Ed.) Marcel Dekker, New York, 1999, Chapter 2, pp. 41-124.

7. J. Felix, P. Gatenholm, Nordic Pulp Paper Res. J. 8, 200 (1993) 